\title{
POPPER, LA NOCIÓN ABSOLUTA DE VERDAD \\ Y EL RELATIVISMO
}

Luis FERNANDEZ MORENO

Dpto. de Filosofía y Ciencias de la Educación

Universidad de Lén.

\section{Introducción: verdad y relativismo}

Uno de los hilos conductores de la obra de Karl Popper radica en su oposición al relativismo, al que consideró como "la principal enfermedad filosófica de nuestro tiempo".' Popper se ocupó más frecuentemente del relativismo epistemológico que del relativismo moral, debido, al menos en parte, a que estimó que el segundo se basaba en buena medida en el primero, por lo que en su enfrentamiento con el relativismo moral Popper hace uso de objeciones dirigidas en primera instancia contra el relativismo epistemológico. En uno de sus escritos más importantes contra el relativismo, [1961], Popper caracteriza el relativismo epistemológico de la siguiente manera:

"Por relativismo [...] entiendo aquí, dicho de manera concisa, la teoría de que la elección entre teorías rivales es arbitraria, puesto que o no existe tal cosa como la verdad objetiva o, en caso de que exista, no hay una teoría que sea verdadera $o$, en todo caso, aunque quizás no sea verdadera - que esté más próxima a la verdad que otra teoría, o si hay dos o más teorías no hay modo de decidir si una de ellas es mejor que otra. ${ }^{\text {"2 }}$

1 Popper [1961], p. 369.

2 Ibid. En este escrito Popper se ocupa con cierta indistinción tanto del relativismo como del escepticismo.

Endoxa: Series Filosoficas, $n^{2} 5,1995$, UNED, Madrid:

Luis Fernández Moreno: Popper, la noción absoluta de verdad y el relativismo. pp. $75-90$. 
Frente a esta posición Popper mantuvo que la elección entre teorías rivales no es arbitraria, pues disponemos de procedimientos para decidir si una teoría es mejor que otra, ${ }^{3}$ hay teorias que están más próximas a la verdad que otras y, por ende, la verdad existe y, más aún, es absoluta $u$ objetiva, rasgos de la verdad que Popper frecuentemente tendió a identificar, como cuando en otro lugar caracteriza el relativismo epistemológico simplemente como "la concepción de que no hay una verdad absoluta u objetiva." ${ }^{4}$ Es fundamentalmente en tomo a la cuestión acerca de si la verdad es o no absoluta donde en opinión de Popper se decide la disputa acerca del relativismo epistemológico.

En este artículo voy a ocuparme de la noción absoluta de verdad mantenida por Karl Popper frente al relativismo epistemologico, para lo que habré de atender también a la teoría tarskiana de la verdad, pues Popper afirma que ha sido Tarski el que ha restablecido esta noción de verdad absoluta u objetiva:

"[La] teoría de la verdad absoluta u objetiva ha sido restablecida por Alfred Tarski."

Popper hace esta afirmación porque considera que el carácter absoluto u objetivo de la noción de verdad viene vinculado a la noción de verdad como correspondencia y él estima que Tarski ha rehabilitado la teoría de la verdad como correspondencia.

A este respecto conviene señalar que Popper fue siempre un partidario de la teoría de la verdad como correspondencia, si bien hasta 1935 se

3 Estos procedimientos se basan fundamentalmente en el "criterio de satisfactoriedad potencial relativa o de progresividad potencial" (Popper [1963], p. 217), que, en resumidas cuentas, establece que una teoría es potencialmente preferible a otra si posee un mayor contenido empírico, donde por "contenido empírico" de una teoría se entiende el conjurto de los falsadores potenciales de la teoría, es decir, el conjunto de los enunciados observacionales que contradicen la teoría (Popper [1963], p. 385 y [1974a], p. 158, nota 16); una teoría es efectivamente, y no sólo potencialmente, mejor que otra si, además de ser potencialmente preferible, supera las contrastaciones severas a que es sometida.

4 Popper [1976], p. 33. Sobre esta identificación del carácter absoluto y objetivo de la verdad consúltese el apartado 3 infra.

5 Popper [1976], p. 35. Véase también Popper [1963], pp. 223 ss. y [1984], pp. 92 s. 
mostró cauteloso en el empleo de la noción de verdad, pues se sentra incapaz de contestar a las objeciones presentadas contra la noción de verdad y, en especial, contra la teoría de la verdad como correspondencia. En opinión de Popper, la objeción fundamental contra la teoría de la verdad como correspondencia consistía en que no se había explicado en qué consiste la correspondencia entre enunciados y hechos; ${ }^{6}$ la elucidación de esta noción de correspondencia es lo que se requeriría fundamentalmente para rehabilitar la teoria de la verdad como correspondencia.

A partir de 1935 Popper empleo sin vacilaciones la noción de verdad; esto se debe a sus encuentros en dicho año con Alfred Tarski y a su conocimiento de la teoría tarskiana de la verdad. Popper sostiene que esta teoría ha rehabilitado definitivamente la teoría de la verdad como correspondencia y que la noción de verdad como correspondencia es una nocion de verdad absoluta u objetiva:

"Denominamos a un enunciado 'verdadero' si corresponde con los hechos [...]. Este es el denominado concepto absoluto u objetivo de verdad [...] Considero la rehabilitación de esta noción de verdad llevada a cabo por el lógico y matemático Alfred Tarski como el resultado filosóficamente más importante de la lógica matemática contemporánea."

En otro lugar Popper afirma:

"El mayor logro de Tarski y la auténtica importancia de su teoría para la filosofía de las ciencias empíricas radica en que él rehabilitó la teoría de la verdad absoluta u objetiva como correspondencia." 8

\footnotetext{
6 Véase Popper [1963], p. 223, [1972], p. 320 y [1979], pp. XXII s. En este artículo voy a seguir a Popper y a Tarski en usar como equivalentes las expresiones "enunciado" y "oración" - en el sentịdo de oración declarativa.

7 Popper [1984], pp. 92 s.

8 Popper [1963], p. 223.
} 
A este respecto cabe plantear varias cuestiones. En primer lugar, si la teoría tarskiana de la verdad puede considerarse como una teoría de la verdad como correspondencia. En segundo lugar, en caso de que la respuesta a esta primera pregunta sea positiva, hay que examinar si la noción de correspondencia con los hechos empleada por Popper ha sido restablecida por la teoría tarskiana. Por último, hay que indagar si la noción tarskiana de verdad posee los rasgos que Popper atribuye a la noción absoluta de verdad y si puede servir, como Popper pretende, de remedio contra el relativismo epistemológico.

\section{Popper, Tarski y la teoría de la verdad como correspondencia}

Para dilucidar si la teoría de la verdad formulada por Alfred Tarski es una teoría de la verdad como correspondencia hay que especificar primero qué entendemos por "teoría de la verdad como correspondencia", pues esta expresión ha sido interpretada de múltiples maneras. Voy a permitirme adoptar en lo siguiente una caracterización de la teoría de la verdad como correspondencia frecuente en la bibliografía sobre teorías de la verdad y que es un tanto minimalista, razón por la cual es difícilmente objetable. Por "teoría de la verdad como correspondencia" voy a entender una teoría que explica la noción de verdad - al menos, la noción de verdad empírica - en base a una relación o a relaciones entre entidades lingǘrticas $\mathrm{y}$ entidades extralingǘrsticas, entre lenguaje y mundo. Precisamente, la relación o las relaciones en cuestión constituirían o explicarían la relación de correspondencia.

Para dilucidar si la teoría tarskiana es en este sentido una teoría de la verdad como correspondencia hay que examinar las dos caracterizaciones de la noción de verdad presentes en dicha teoría, a saber, la definición de verdad como satisfacción por toda secuencia de objetos y las equivalencias de la forma (V) - o, más brevemente, equivalencias (V). Estas equivalencias son obtenidas a partir del esquema de oración (V), "X es verdadera si y sólo si $\mathrm{p}$ ", mediante la sustitución de " $\mathrm{X}$ " por el nombre metalingüístico de una oración del lenguaje-objeto y de "p" por la traducción metalingüística de dicha oración; un ejemplo de equivalencia (V) es "La oración 'El Everest es una montaña' es verdadera si y sólo si 
el Everest es una montaña". 9 Tarski considera a cada equivalencia (V) como una definición parcial de la verdad con respecto a un lenguaje-objeto determinado, pues cada una de estas equivalencias proporciona condiciones necesarias y suficientes para la verdad de una oración del lenguaje-objeto, y establece como condición de adecuación extensional de una definición de verdad que de tal definición han de derivarse todas las equivalencias (V) formadas con las oraciones del lenguaje-objeto en cuestion; Tarski muestra que su definición de verdad mediante satisfacción cumple esta condición de adecuación extensional. Por tanto, hemos de preguntarnos si la definición tarskiana de verdad mediante satisfacción y las equivalencias (V) pueden considerarse como componentes de una teoría de la verdad como correspondencia. Mi respuesta a estas dos cuestiones será positiva.

En primer lugar, la definición de verdad mediante satisfacción puede considerarse efectivamente como una definición de verdad conforme con la teoría de la verdad como correspondencia, pues la noción de satisfacción expresa una relación entre un tipo de entidades lingüísticas como son las funciones sentenciales - incluido el caso límite de las mismas, es decir, las oraciones - y entidades del mundo, a saber, objetos o secuencias de objetos. Incluso si se concede que las secuencias de objetos no son en sentido estricto entidades del mundo, sino abstracciones obtenidas a partir de las mismas, esto no es óbice para que en base a los objetos integrantes de tales secuencias la noción de verdad sea explicada mediante una relación entre lenguaje y mundo; esto es justamente lo característico de una teoría de la verdad como correspondencia. En este sentido la definición de verdad mediante satisfacción formulada por Tarski puede ser considerada como integrante de una teoría de la verdad como correspondencia.

Por otra parte, las equivalencias (V) pueden interpretarse también como componentes de una teoría de la verdad como correspondencia, pues la oración que constituye el miembro derecho de dichas equivalen-

9 En la formulación de esta equivalencia se presupone que el metalenguaje contiene al lenguaje-objeto como parte. En lo siguiente supondré que el metalenguaje cumple esta condición. 
cias nos dice cómo ha de ser el mundo para que la oración cuyo nombre aparece como sujeto de su miembro izquierdo sea verdadera; por ejemplo, la equivalencia (V) "la oración 'El Everest es una montaña' es verdadera si y sólo si el Everest es una montaña" nos dice que la oración "El Everest es una montaña" es verdadera si y sólo si el mundo es de tal manera que el Everest es una montaña. Por consiguiente, una teoría de la verdad como correspondencia puede aceptar las equivalencias (V) como dando condiciones de verdad necesarias y suficientes y, por tanto, como definiciones parciales de la noción de verdad.

Esta justificación de la conformidad de las equivalencias (V) con la teoría de la verdad como correspondencia puede tomarse más explícita, si tenemos en cuenta que la interpretación de los signos del lenguajeobjeto y, por tanto, también de los signos del metalenguaje es su interpretación referencial usual, es decir, las constantes de individuo denotan individuos, las constantes de predicado denotan conjuntos o tuplos de individuos - expresado de manera informal, propiedades o relaciones entre individuos -, etc. De esta manera las equivalencias (V) pueden interpretarse en el sentido de que la verdad de las oraciones cuyos nombres constituyen los sujetos de los miembros izquierdos de dichas equivalencias depende de las propiedades de los individuos, de las relaciones entre los individuos, etc. expresadas por dichas oraciones; por tanto, la verdad de cada oración del lenguaje-objeto depende de cómo es el mundo, es decir, depende de si el mundo es como afirma dicha oración. En este sentido las equivalencias (V) pueden interpretarse de acuerdo con la teoría de la verdad como correspondencia.

Cabría replicar, no obstante, que, si bien las equivalencias (V) pueden interpretarse de manera que la verdad de las oraciones depende de como es el mundo, en ellas no se apela a relaciones entre lenguaje y mundo. Pero a este respecto cabe sefialar que, aunque tales relaciones no aparecen de manera explícita en dichas equivalencias, si se apela a ellas en la interpretación de las mismas, por la sencilla razón de que tales relaciones subyacen a la interpretación de las constantes primitivas del lenguajeobjeto y, por tanto, también del metalenguaje que figuran en dichas equivalencias, por ejemplo, la relación de denotación para constantes de individuo, la relación de denotación o aplicación para predicados, etc. De 
esta manera el que la oración "El Everest es una montaña" sea verdadera si y sólo si el Everest es una montaña se debe a que la expresión "El Everest" denota el Everest y a que el predicado "es una montaña" denota el conjunto de las montañas o se aplica a los objetos que son montañas.

Por consiguiente, podemos concluir que tanto la definición de verdad mediante satisfacción como las equivalencias $(V)$ pueden interpretarse en un sentido acorde con la teoría de la verdad como correspondencia. El que la teoria tarskiana de la verdad pueda considerarse como una teoría de la verdad como correspondencia concuerda muy posiblemente con el espíritu que guio a Tarski en la formulación de su teoria, pues Tarski se propuso formular una teoría que constituyese una precisión de la concepción clásica de la verdad, a la que identificó explícitamente en sus primeras obras con la teoría de la verdad como correspondencia. ${ }^{10}$

Pero, aunque la teoría tarskiana pueda ser considerada como una teoría de la verdad como correspondencia, en ella no aparece la noción de verdad como correspondencia con los hechos empleada por Popper y cuya elucidación él atribuye a Tarski. Popper cree que la relación de correspondencia entre oraciones y hechos viene expresada por las equivalencias $(V)$, pues él presupone que las oraciones declarativas y, por tanto, los miembros derechos de las equivalencias (V) describen hechos (reales o meramente posibles) y, además, se permite sustituir en estas equivalencias el predicado "verdadero" por la expresión "corresponde con los hechos" o "corresponde con la realidad". Por lo tanto, una equivalencia (V) como "La oración 'El Everest es una montaña' es verdadera si y sólo si el Everest es una montaña" afirmaría según Popper que la oración "El Everest es una montaña" corresponde con los hechos si y sólo si el hecho descrito por dicha oración es un hecho real, es decir, si y sólo si el que el Everest es una montaña es un hecho real.

La interpretación que Popper presenta de las equivalencias (V) plantea varios problemas; los dos principales son los siguientes. En primer lugar, suponiendo que haya algo así como hechos posibles, Popper no ha presentado ningún criterio para distinguir entre hechos reales y hechos posibles, limitándose básicamente a indicar que los primeros son descritos

10 Véase Tarski [1935], p. 265 y [1936], p. 1. 
por oraciones verdaderas, ${ }^{11}$ pero el recurso a este proceder en la elucidación de la noción de verdad como correspondencia con los hechos es obviamente circular. En segundo lugar, Tarski mantuvo la neutralidad ontológica de su teoría de la verdad, razón por la cual esta teoría podría ser aceptada por cualesquiera posiciones ontológicas; por tanto, toda la carga ontológica de carácter realista con la que Popper suplementa la teoría tarskiana de la verdad y, en especial, las equivalencias (V) le es completamente extraña a la teoría tarskiana.

Por consiguiente, aunque cabe considerar a la teoría tarskiana de la verdad como una teoría de la verdad como correspondencia, dicha teoría no rehabilita de ninguna manera la noción de verdad como correspondencia en el sentido que Popper pretende, es decir, la noción realista de correspondencia con los hechos. No obstante, cabe preguntarse si la noción tarskiana de verdad es una noción absoluta de verdad. La respuesta a esta pregunta dependerá a su vez de lo que se entienda por el carácter absoluto de la verdad. Esto nos lleva a ocuparnos, en primer lugar, de en qué sentido considera Popper que la verdad es absoluta. Posteriormente atenderé a la cuestión de si algunas de las acepciones de la noción absoluta de verdad en Popper están presentes en la teoría tarskiana de manera que ésta pueda considerarse, como Popper afirma, como un antídoto contra el relativismo epistemológico.

\section{La noción absoluta de verdad en Karl Popper}

En la obra de Popper cabe distinguir diversas acepciones de la noción absoluta de verdad, pero las dos más importantes son las que aparecen mencionadas en el siguiente texto:

"la verdad es absoluta, es decir, no es relativa, digamos, al tiempo

o a lo que la gente piense acerca de ella." ${ }^{12}$

11 Popper [1972], p. 329

12 Popper/Eccles [1974], p. 89. Popper también considera que la verdad es absoluta en el sentido de que no es relativa a un lenguaje; sobre esta acepción de la absolutez de la verdad consúltese el apartado 4 infra. 
De esta manera Popper mantiene, por una parte, que la verdad no es relativa al tiempo, la verdad es intemporal. Popper ha formulado esta tesis en otros lugares con mayor contundencia:

"Si un enunciado formulado sin ambigüedad es verdadero ahora, entonces es verdadero por siempre, y siempre será verdadero: la verdad es intemporal ( $y$ así también la falsedad)."13

Por otra parte, la verdad es absoluta porque la verdad o falsedad de un enunciado no depende de nuestra opinión al respecto y, por tanto, no es relativa a nuestras creencias. ${ }^{14}$ Este último sentido de la absolutez de la verdad constituye al mismo tiempo una acepción en la que Popper considera que la verdad es objetiva, lo que se observa cuando Popper viene a identificar el carácter objetivo de la verdad con su carácter impersonal. ${ }^{15}$ Puesto que Popper se refiere en numerosas ocasiones a la verdad como "absoluta u objetiva", ${ }^{16}$ es de suponer que en tales contextos él entiende ei carácter absoluto de la verdad fundamentalmente en el segundo sentido mencionado, que Popper resume diciendo que "la verdad está por encima de toda autoridad humana."17

Cabe indicar que en la obra de Popper parece haber al menos otra acepción de la objetividad de la verdad: la verdad es objetiva en el sentido de que es una propiedad de entidades del mundo 3 y ella misma es un habitante del mundo $3 .^{18}$

Como es sabido, Popper considera que la realidad - o el mundo consta de tres submundos ontologicamente distintos: el mundo 1 o mundo

13 Popper [1974a], p. 148. Este carácter intemporal de las nociones de verdad y falsedad fue mantenido por Popper ya en [1935], p. 220.

14 Popper [1974a], p. 114.

15 Popper [1963], p. 375.

16 A este respecto pueden consultarse los pasajes de las notas $4,5,7$ y 8 , así como Popper [1974b], pp. 1002, 1114 y 1157.

17 Popper [1963], p. 29.

18 Véase [1974a], pp. 114 y 155, así como [1972], p. 158. 
físico, el mundo 2 o mundo mental y el mundo 3 o mundo de los contenidos objetivos de pensamiento, especialmente de los pensamientos científicos y poéticos y de las obras de arte. ${ }^{19} \mathrm{El}$ contenido de las teorías y de los enunciados pertenece así al mundo 3 y lo mismo le ocurre a esa propiedad de tales entidades que es su verdad o falsedad.

No voy a detenerme aquí en esta acepción de la objetividad de la verdad, pero quiero hacer dos observaciones al respecto. En primer lugar, Popper parece introducir esta segunda acepción de la objetividad de la verdad para afianzar la objetividad de la verdad en su primera acepción y, por tanto, el carácter absoluto de la verdad en el segundo sentido mencionado -, pero postular la existencia del mundo 3 para consolidar la absolutez u objetividad de la verdad en el sentido de que si un enunciado es verdadero o falso no depende de nuestras creencias al respecto, es, aparte de innecesario, contraproducente. Es innecesario porque para preservar la absolutez u objetividad de la verdad en este sentido no se requiere situar la verdad en un mundo distinto al que habitamos los mortales - mundos 1 y 2 -, y es contraproducente porque la postulación del mundo 3 nos involucra de inmediato en innumerables dificultades, concemientes, por ejemplo, a las relaciones de los mundos 1 y 2 con el mundo 3 o a la parcial autonomía del mundo 3, a las que ni Popper ni ninguno de sus seguidores ha respondido hasta ahora de manera mínimamente satisfactoria. En segundo lugar, es obvio de antemano que esta segunda acepción de la objetividad de la verdad no puede tener ninguna relación con los rasgos de la noción de verdad que un autor con inclinaciones nominalistas como Tarski haya podido mantener.

Retomando el carácter absoluto de la verdad, tal como Popper lo entiende, conviene señalar que Popper complementa sus observaciones al respecto añadiendo que, aunque la verdad sea absoluta en los dos sentidos mencionados, carecemos de un criterio general de verdad que nos permita justificar la verdad o falsedad de nuestros enunciados. En relación con este aspecto de la cuestión Popper alude a uno de los teoremas demostra-

19 Los escritos de Popper en los que se presenta de manera más detallada su teoría de los tres mundos son [1972] y Popper/Eccles [1977]. Acerca de algunas de las dificultades involucradas en las afirmaciones de Popper acerca del mundo 3 puede consultarse Cohen [1980]. 
dos por Tarski, apoyándose en resultados obtenidos por Gödel, según el cual para todo lenguaje que sea suficientemente potente como para formular la aritmética de los números naturales hay enunciados verdaderos cuya verdad no puede demostrarse en dicho lenguaje. ${ }^{20}$ Popper interpreta este resultado en el sentido de que hay que distinguir tajantemente entre las nociones de verdad y certeza; la certeza o seguridad absoluta en la posesión de la verdad nos está vedada, sobre todo por lo que respecta al ámbito de las ciencias empíricas.

De esta manera el carácter absoluto de la verdad viene complementado por nuestra falibilidad, ${ }^{21}$ si bien esto no es óbice para que podamos ofrecer buenas razones acerca de la verdad o falsedad de un enunciado. Nuestra falibilidad no se opone a que nuestras teorías puedan ser verdaderas, ni a que podamos ofrecer buenas razones en apoyo de su verdad, sino sólo a que podamos dar razones concluyentes, a que podamos estar seguros de su verdad y a que no podamos equivocarnos. En este sentido la noción absoluta de verdad desempeña en la epistemología de Karl Popper el papel de una idea regulativa que guia la investigación cientifica.

El carácter absoluto de la verdad unido al falibilismo - Popper caracteriza su posición como un "absolutismo falibilista"22 - nos permite hacer afirmaciones como las siguientes:

"Una teoría puede ser verdadera incluso aunque nadie la crea, e incluso aunque no tengamos razones para pensar que es verdadera, y otra teoria puede ser falsa incluso aunque tengamos comparativamente buenas razones para aceptarla." ${ }^{23}$

\footnotetext{
20 Véase Tarski [1935], pp. 400 ss.

21 Popper considera que la ausencia de un criterio general de verdad es "la razón más profunda de la falibilidad humana" (Popper/Eccles [1974], p. 88).

22 Popper [1961], p. 377.

23 Popper [1963], p. 225.
} 


\section{Popper, Tarski y la noción absoluta de verdad}

Una vez que hemos examinado en qué sentido considera Popper que la verdad es absoluta, y puesto que él estima que Tarski ha rehabilitado esta noción absoluta de verdad, hemos de atender a la teoría tarskiana para determinar si en ella encontramos algún rastro de los rasgos que Popper incluye en el carácter absoluto de la verdad. ${ }^{24}$

Pero conviene indicar previamente que hay un sentido en el que Tarski ha afirmado que la noción de verdad es relativa. Tarski considera como portadores primarios de verdad a las oraciones, pero la noción de oración es relativa a un lenguaje, pues una expresión que en un lenguaje es una oración bien formada, puede ser en otro lenguaje mal formada o carente de sentido. Por tanto, en sentido estricto, Tarski no ha definido el predicado "verdadero", ni siquiera la expresión "oración verdadera", sino la expresion "oración verdadera en L", donde "L" es un lenguajeobjeto que posee una estructura exactamente especificada y que no es semánticamente cerrado, es decir, que no contiene sus propios términos semánticos, pues, en otro caso, nos veriamos involucrados en paradojas semánticas. La noción de verdad es tan relativa a un lenguaje como la noción de oración. ${ }^{25}$ Popper reconoce que Tarski ha afirmado la relatividad de la noción de verdad en tal sentido; ${ }^{26}$ sin embargo, él considera que dicha relatividad es neutralizada por el hecho de que si una oración de un lenguaje es verdadera, su traducción a otro lenguaje también lo será:

"[...] la verdad no es una noción relativa a un lenguaje, pues si $\mathbf{P}_{1}$

es un enunciado de un lenguaje $L_{1}$ y $P_{2}$ un enunciado de un

24 Como ha señalado Haack ([1978], p. 115), Popper no presta atención al hecho de que la definición de verdad formulada por Tarski en [1935] lo es de una noción absoluta de verdad, por oposición a la noción de verdad relativa a un modelo o a una estructura, que Tarski no definió hasta la década de los cincuenta. Sin embargo, no creo que Popper hubiera otorgado importancia alguna al hecho de que la noción tarskiana de verdad fuese absoluta en este sentido.

25 Tarski [1944], p. 342.

26 Popper [1976], p. 35. 
lenguaje $\mathrm{L}_{2}$, entonces se cumple lo siguiente (digamos, en $\mathrm{L}_{\mathrm{m}}$ ): si $P_{2}$ es una traducción de $P_{1}$ de $L_{1}$ en $L_{2}$, entonces $P_{1}$ y $P_{2}$ han de ser 0 ambos verdaderos o ambos falsos, es decir, han de tener el mismo valor de verdad. ${ }^{27}$

Popper tiene obviamente razón en que si una oración de un lenguaje es verdadera su traducción a otro lenguaje también lo será, si bien, como nos recordo Quine hace décadas, la noción de traducción es bastante más compleja de lo que Popper parece suponer. En cualquier caso, el sentido mencionado en el que Tarski afirma la relatividad de la noción de verdad es trivial e irrelevante en la disputa acerca del relativismo epistemológico.

Por lo que respecta a si la noción de verdad es intemporal o, si por el contrario, es relativa al tiempo, Tarski sólo se expresó de manera muy indirecta. En un pasaje del parágrafo 21 de [1944], antes de atender a las relaciones entre la noción de aceptabilidad de teorías y la noción de verdad, Tarski hace hincapié en la relatividad de la noción de aceptabilidad:

"Esta noción de aceptabilidad ha de ser relativizada a una etapa dada del desarrollo de una ciencia (o a una porción dada de conocimiento presupuesto). En otras palabras, podemos considerarla provista de un coeficiente temporal, pues una teoría que es aceptable hoy puede tomarse insostenible mañana como resultado de nuevos descubrimientos científicos. ${ }^{28}$

Tarski examina a continuación dos requisitos concernientes a la aceptabilidad de teorias que involucran la noción de verdad, y del contexto en cuestion ${ }^{29}$ resulta manifiesto que Tarski considera que las nociones de verdad y de falsedad, a diferencia de la noción de aceptabilidad, carecen de dicha relatividad temporal. Es cieno que esta opinión de Tarski no forma parte en sentido estricto de su teoría de la verdad, la cual consta

\footnotetext{
27 Popper [1972], p. 45. Véase también [1976], p. 35.

28 Tarski [1944], p. 366.

29 Tarski [1944], pp. 367 s.
} 
de los requisitos materiales y formales que ha de cumplir la definición de verdad, de esta definición y de los teoremas derivables de la misma, pero también lo es que en ninguno de estos componentes de la teoría tarskiana de la verdad aparece factor alguno que pudiese implicar la relatividad temporal de la noción de verdad.

Por último, en sus escritos sobre semántica Tarski no hizo ninguna observación, ni siquiera indirecta, acerca de si él considera que la noción de verdad es absoluta en el sentido de que la verdad de un enunciado no es relativa a nuestras creencias al respecto, si bien, como ocurría en el caso anterior, ninguno de los componentes de su teoría de la verdad conlleva tal relatividad.

Ahora bien, el hecho de que en la teoría tarskiana no aparezca ningún factor que implique la relatividad de la verdad o que impida que la noción de verdad sea absoluta en el sentido en el que Popper entiende el carácter absoluto de la verdad -exceptuando su relatividad a un lenguaje-, no conlleva necesariamente que dicha teoría defienda una noción absoluta de verdad $y$, por tanto, que dicha teoría sea incompatible con el relativismo epistemológico, como Popper pretende al hacer afirmaciones como las siguientes:

"La verdad es objetiva y absoluta; ésta es la idea que Alfred Tarski ha defendido contra el relativismo."

"Me gustaría indicar brevemente por qué no soy un relativista: creo en la verdad 'absoluta' y 'objetiva' en el sentido de Tarski $[\ldots]^{31}$

Aunque Tarski consideró su teoría como una versión actualizada y más precisa de la teoría de la verdad como correspondencia, también hizo hincapié al mismo tiempo en la neutralidad epistemológica de su teoría de la verdad, que incluye, por tanto, su neutralidad con respecto al

30 Popper [1972], p. VII.

31 Popper [1970], p. 56. En este texto la expresión "absoluto" - y lo mismo cabría decir con respecto a la expresión "objetivo" - aparece entrecomillada posiblemente para dar a entender que el carácter absoluto de la verdad no conlleva que estemos en posesión de ella y que podamos hablar con absoluta certeza, sino más bien todo lo contrario. 
relativismo epistemológico. Tarski, quien se refirió a su teoría de la verdad en [1944] como "la concepción semántica de la verdad", afirma en este artículo:

"[...] podemos aceptar la concepción semántica de la verdad sin abandonar ninguna posición epistemológica que podamos haber tenido. Podemos seguir siendo realistas ingenuos, realistas críticos o idealistas, empiristas o metafísicos - lo que hayamos sido antes. La concepción semántica es completamente neutral con respecto a todas estas posiciones." 32

La neutralidad epistemológica de la teoría tarskiana de la verdad - y lo mismo es aplicable a su neutralidad ontológica - se basa en que esta teoría no se compromete con una determinada posición acerca de las condiciones bajo las cuales una oración "p" puede ser afirmada o aceptada; la teoría tarskiana implica sólo que, siempre que afirmemos o rechacemos la oración " $\mathrm{p}$ ", hemos de estar dispuestos a afirmar o rechazar la oración "'p' es verdadera". ${ }^{33}$ Pero si esto es así, la teoría tarskiana de la verdad no es incompatible con cl relativismo epistemologico. El relativista mantendrá que las condiciones para afirmar "p" son relativas al tiempo, a nuestras creencias, etc. y cuando en base a dichas condiciones él afirme la oración " $\mathrm{p}$ ", afirmará también la oración "'p' es verdadera". ${ }^{34}$ De esta manera el relativismo epistemologico es compatible con la teoría tarskiana y, por consiguiente, la tesis mantenida por Karl Popper acerca de que la teoría tarskiana de la verdad ha restablecido una noción absoluta de verdad y constituye el remedio contra el relativismo epistemologico carece de fundamento.

\footnotetext{
32 Tarski [1944], p. 362.

33 Tarski [1944], p. 361.

34 A este respecto son asimismo dignas de consideración las observaciones de Kuhn en [1970], pp. 265 s. sobre las apelaciones a Tarski por parte de Popper.
} 


\section{Bibliografía}

Cohen, J.L. [1980]: "Some Comments on Third World Epistemology". British Journal for the Philosophy of Science, 31, pp. 175-180.

Haack, S. [1978]: Philosophy of Logics. Cambridge: Cambridge University Press.

Kuhn, T. [1970]: "Reflections on my Critics". En Lakatos/ Musgrave, pp. 231-278.

Lakatos, I. y A. Musgrave (eds.) [1970]: Criticism and the Growth of Knowledge. Londres: Cambridge University Press.

Popper, K. [1935]: Logik der Forschung. Tubinga: Mohr; 8' ed. rev. y ampliada, 1984.

[1945]: The Open Society and Its Enemies. 2 vols. Londres: Routledge \& Kegan Paul; $4^{2}$ ed. rev. y ampliada, 1962.

[1961]: "Facts, Standards, and Truth: A Further Criticism of Relativism". Incluido como apéndice al segundo volumen de la edición de 1962 de Popper [1945], pp. 369-396.

[1963]: Conjectures and Refutations. The Growth of Scientific Knowledge. Londres: Routledge \& Kegan Paul; 4" ed. rev., 1979.

[1970]: "Normal Science and Its Dangers". En Lakatos/ Musgrave, pp. 51-58.

[1972]: Objective Knowledge. An Evolutionary Approach. Oxford: Oxford U.P.; 4" ed. rev. y ampliada, 1985.

[1974a]: "Intellectual Autobiography". En Schlipp [1974], pp. 3-181.

[1974b]: "Replies to my Critics". En Schlipp [1974], pp. 961-1197.

[1976]: "The Myth of the Framework". En E. Freeman (ed.), The Abdication of Philosophy. Philosophy and the Public Good, La Salle, Open Court, pp. 23-48.

[1979]: Die beiden Grundprobleme der Erkenntnistheorie. Tubinga: Mohr.

[1983]: Realism and the Aim of Science. Totowa: Rowman \& Littlefield.

[1984]: Auf der Suche nach einer besseren Welt. Vorträge und Aufsätze aus dreißig Jahren. Munich: Piper.

Popper, K y J. Eccles [1974]: "Falsifiability and Freedom". En F. Elders et al. (eds.), Reflexive Water. The Basic Concerns of Mankind, Londres, Souvenir Press, pp. 71-131.

[1977]: The Self and Its Brain. Berlin: Springer.

Schlipp, P.A. (ed.) [1974]: The Philosophy of Karl Popper. 2 vols. La Salle: Open Court. Tarski. A. [1935]: "Der Wahrheitsbegriff in den formalisierten Sprachen". Studia Philosophica, 1, pp. $261-405$.

[1936]: "Grundlegung der wissenschaftlichen Semantik". Actes du Congrès International de Philosophie Scientifique. París: Hermann, vol. 3, pp. 1-8.

[1944]: "The Semantic Conception of Truth and the Foundations of Semantics". Philosophy and Phenomenological Research, 4, pp. 341-375. 